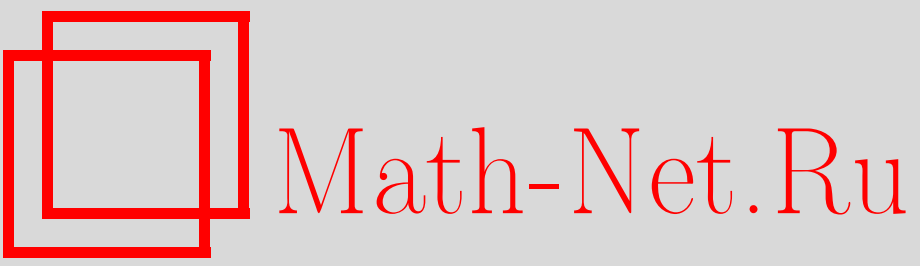

Л. Ц. Аджемян, А. Н. Васильев, Н-модель критической динамики: выбор динамических переменных, исключение звуковых мод, уравнения для звуковых волн вблизи $T_{\mathrm{c}}, T M \Phi, 1998$, том 117, номер 1, 140-160

DOI: https://doi.org/10.4213/tmf923

Использование Общероссийского математического портала Math-Net.Ru подразумевает, что вы прочитали и согласны с пользовательским соглашением

http://www.mathnet.ru/rus/agreement

Параметры загрузки:

IP : 3.95.254.165

26 апреля 2023 г., 13:04:26 


\author{
ТЕОРЕТИЧЕСКАЯ \\ И МАТЕМАТИЧЕСКАЯ \\ ФИЗИКА \\ Том 117, № 1 \\ октябрь, 1998
}

(C) 1998 г.

Л.Ц. Аджемян*, А.Н. Васильев*

\title{
Н-МОДЕЛЬ КРИТИЧЕСКОЙ ДИНАМИКИ: ВЫБОР ДИНАМИЧЕСКИХ ПЕРЕМЕННЫХ, ИСКЛЮЧЕНИЕ ЗВУКОВЫХ МОД, УРАВНЕНИЯ ДЛЯ ЗВУКОВЫХ ВОЛН ВБЛИЗИ $T_{\mathrm{c}}$
}

\footnotetext{
Обсуждается полная флуктуационная модель, описывающая динамику всех мягких мод в окрестности критической точки фазового перехода жидкость-газ.
}

\section{1. ВВЕДЕНИЕ}

Настоящая статья открывает серию работ, посвященных ревизии известной Н-модели [1-3]. Н-модель используется при описании критической динамики в окрестности точки фазового перехода жидкость-газ для простых жидкостей и аналогичной критической “точки расслоения" в бинарных смесях. Эти две задачи на уровне феноменологии различаются, и для определенности мы будем говорить лишш о критической точке перехода жидкость-газ в простых жидкостях с молекулами одного сорта.

В этой статье обсуждаются проблема выбора динамических переменных Н-модели и процедура исключения звуковых мод из соответствующих стохастических уравнений. Приводится также вывод уравнений для звуковых волн и соответствующего закона дисперсии в критической области.

Считается [1-3], что роль основного поля параметра порядка $\psi$ в Н-модели играет величина $\Delta \bar{S}=\bar{S}-\bar{S}_{\text {c }}$, где $\bar{S}=S / \rho$ - “энтропия на одну частицу" (кратная "энтропии на единицу массы"), а $S$ и $\rho$ (здесь и далее) - объемные плотности энтропии и числа

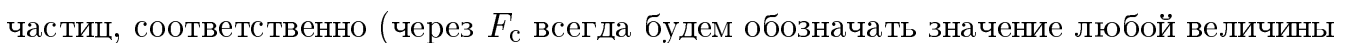
$F$ в критической точке, $\Delta F \equiv F-F_{\mathrm{c}}$ ). Основанием для такого выбора является то, что именно для величины $\Delta \bar{S}$ в линеаризованных уравнениях гидродинамики идеальной (т.е. в пренебрежении эффектами теплопроводности и вязкости) жидкости имеет место отщепление от продольной компоненты поля скорости, связанной со звуковыми колебаниями. Действительно, точные уравнения гидродинамики идеальной жидкости для обеих величин $F=\rho, S$ и поля скорости $v_{i}$ имеют вид [4] $\partial_{t} F+\partial_{i}\left(v_{i} F\right)=0$ (по

\footnotetext{
* Санкт-Петербургский государственный университет, Санкт-Петербург, Россия
} 
повторяющимся индексам всегда подразумевается суммирование), откуда для соответ-

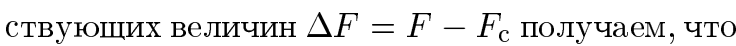

$$
\partial_{t} \Delta F+F_{\mathrm{c}} \partial_{i} v_{i}+\partial_{i}\left(v_{i} \Delta F\right)=0, \quad F=\rho, S
$$

Из уравнения (1) видно, что в линейном по совокупности “малых величин" $\Delta F, v$ приближении (когда последним слагаемым в левой части (1) можно пренебречь) вклад с продольной составляющей поля скорости $\partial_{i} v_{i}$ исчезает для той линейной комбинации $\Delta \rho$ и $\Delta S$, которая в линейном приближении кратна величине

$$
\Delta \bar{S}=\rho_{\mathrm{c}}^{-2}\left[\rho_{\mathrm{c}} \Delta S-S_{\mathrm{c}} \Delta \rho\right]
$$

Именно поэтому $\Delta \bar{S}$ обычно и принимают за основное поле параметра порядка, считая при этом, что соответствующая критическая статика описывается простой $\psi^{4}$-моделью Гинзбурга-Ландау-Вильсона (критическая точка перехода жидкость-газ относится к классу эквивалентности модели Изинга). При этом игнорируется тот факт, что комбинация (2) в критической статике является в действительности смесью двух слагаемых с разной критической размерностью: одного - с размерностью самого поля $\psi$ в $\psi^{4}$-модели, другого - с размерностью составного оператора $\psi^{2}$. Хотя это объективньй и допускающий экспериментальную проверку факт, при построении Н-модели его обычно не принимают во внимание, полагая, что отшепление от $\partial v$ в уравнении типа (1) имеет решающее значение.

Мы покажем, что такая точка зрения ошибочна. "Правильным" полем параметра порядка следует считать не величину (2), а ту линейную комбинацию $\Delta \rho$ и $\Delta S$, которая в статике точно соответствует основному полю $\psi$ и имеет соответствующую критическую размерность $\Delta_{\psi}$. Хотя для этой комбинации сцепление с продольной составляюшей поля скорости остается, в действительности это значения не имеет (вопреки общепринятому мнению), что будет строго доказано в разделе 4 путем анализа процедуры исключения звуковых мод из динамики Н-модели. В конечном итоге мы придем к обычным динамическим уравнениям Н-модели только с иным смыслом поля $\psi$. При этом будет показано, что это изменение не меняет смысла коэффициента Онзагера $\lambda$ (коэффициент теплопроводности) в динамическом уравнении для $\psi$. Этот коэффициент остается точно таким же, как и для поля (2) (т.е. обшепринятым), несмотря на изменение смысла самого поля $\psi$. Важно также, что исходная полная система стохастических уравнений для данной задачи является корректной основой при анализе проблемы распространения звука вблизи критической точки перехода жидкость-газ. Соответствуюшие динамические уравнения для звуковых волн выводятся в разделе 5 .

\section{2. КРИТИЧЕСКАЯ СТАТИКА ПЕРЕХОДА ЖИДКОСТЬ-ГАЗ}

В этом разделе приводятся необходимые для дальнейшего краткие справочные сведения о критической статике перехода жидкость-газ. Этот переход принадлежит классу эквивалентности модели Изинга и описьвается простой $\psi^{4}$-моделью, которой при 
записи весового множителя в виде $\exp S$ соответствует функционал действия $S(\psi)=$ $-(\partial \psi)^{2} / 2-\tau \psi^{2} / 2-g \psi^{4} / 24+h \psi$, где $\tau=T-T_{\mathrm{c}}, h-$ внешнее магнитное поле. По $d$-мерному пространственному аргументу $\mathbf{x}$ поля $\psi$ в статических функционалах и по $x=(t, \mathbf{x})$ в динамических всегда подразумевается интегрирование. Формальным аналогом удельной (в расчете на узел) свободной энергии магнетика $F(T, h)$ для большого канонического ансамбля газа является величина $-p(T, \mu)$, где $p$ - давление, $\mu$ - химический потенциал. Однако аналогия между $h$ и $\mu$ неполная, т.к. на плоскости $T, \mu$ линия сосуществования жидкой и газовой фаз $\mu=\mu_{0}(T), T \leqslant T_{\mathrm{c}}$, имеет конечный наклон вблизи точки ее окончания $T=T_{\mathrm{c}}, \mu=\mu_{\mathrm{c}}=\mu_{0}\left(T_{\mathrm{c}}\right)$ из-за отсутствия характерной для магнетика симметрии относительно перемены знака поля. Поэтому точными аналогами

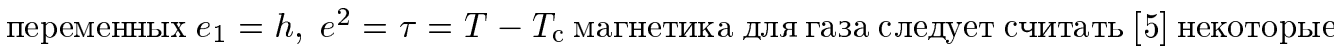
линейные комбинации

$$
e_{1}=\Delta \mu+a \Delta T, \quad e_{2}=\Delta T+b \Delta \mu,
$$

где $a$ и $b$ - числовые коэффициенты. Линия сосуществования фаз вблизи $T_{\text {с }}$ считается прямой $e_{1}=0, T \leqslant T_{\text {c }}$ (ее кривизна, т.е. наличие возможных нелинейностей в соотношениях (3) (поправки к скейлингу), обсуждаться и учитываться не будет). Коэффициент $a$ определяется наклоном этой прямой. Коэффициенты $a$ и $b$ теоретически можно вычислить по статсумме большого канонического ансамбля газа, но нельзя измерить непосредственно (подробнее об этом см. в конце раздела). По правилам термодинамики [6]

$$
d p=S d T+\rho d \mu
$$

В окрестности критической точки

$$
p(T, \mu)=p_{\mathrm{c}}+S_{\mathrm{c}} \Delta T+\rho_{\mathrm{c}} \Delta \mu+p^{(\mathrm{s})},
$$

где первые три слагаемых - регулярная, а последнее слагаемое - сингулярная части давления. Для магнетика аналогично: $F(T, h)=F_{\mathrm{c}}-S_{\mathrm{c}} \Delta T+F^{(\mathrm{s})}$. Согласно идее универсальности функции $F^{(\mathrm{s})}(e)$ и $-p^{(\mathrm{s})}(e)$ для $d$-мерной задачи должны иметь одинаковое скейлинговое представление типа

$$
-p^{(\mathrm{s})} \sim\left|e_{1}\right|^{d / \Delta_{1}} f(z), \quad z \sim e_{2}\left|e_{1}\right|^{\Delta_{2} / \Delta_{1}}
$$

с одинаковыми для обеих задач критическими размерностями $\Delta_{i}=\Delta\left[e_{i}\right]$ переменных $e_{i}$ и одинаковой (с точностью до нормировок) скейлинговой функцией $f$ от критически безразмерного аргумента $z$. При этом для магнетика $e_{1}=h, e_{2}=\tau=\Delta T$, а для газа роль переменных $е$ играют величины (3). В пренебрежении поправками, возникающими от слагаемого $p^{(\mathrm{s})}$, из (5) имеем $\Delta p=S_{\mathrm{c}} \Delta T+\rho_{\mathrm{c}} \Delta \mu$, что позволяет выразить переменные (3) через непосредственно измеримые величины $\Delta T$ и $\Delta p$.

Из соотношений $(3)$ и (4) имеем $\partial_{\mu}=\partial_{1}+b \partial_{2}, \partial_{T}=a \partial_{1}+\partial_{2}\left(\partial_{i} \equiv \partial / \partial e_{i}\right)$ и

$$
\Delta \rho=\left(\partial_{1}+b \partial_{2}\right) p^{(\mathrm{s})}, \quad \Delta S=\left(a \partial_{1}+\partial_{2}\right) p^{(\mathrm{s})} .
$$


Определенные критические размерности имеют величины $p^{(\mathrm{s})}$ и переменные $e$, а величины (6) - суммы двух вкладов с разной размерностью: $d-\Delta_{1}$ для $\partial_{1} p^{(\mathrm{s})}$ (как у намагниченности в модели Изинга) и $d-\Delta_{2}$ для $\partial_{2} p^{(\mathrm{s})}$ (как у сопряженного с $\tau$ оператора $\psi^{2}$ ). Численно $\Delta_{1}>\Delta_{2}$, поэтому операция $\partial_{1}$ "более сингулярна", чем $\partial_{2}$, она и определяет ведущие вклады.

На линии сосушествования фаз $e_{1}=0, \Delta T \leqslant 0$ величины $\partial_{2} p^{(\mathrm{s})}$ однозначны, а $\partial_{1} p^{(\mathrm{s})}$ двузначны. Эти величины исчезают при $e_{1}=0, \Delta T>0$. Отсюда следует, что на линии $e_{1}=0$ величины $\Delta \rho$ и $\Delta S$ при $\Delta T>0$ однозначны и имеют асимптотику $\Delta \rho \equiv \rho-\rho_{\mathrm{c}} \cong$ $b A_{+}\left|e_{2}\right|^{1-\alpha}, \Delta S \equiv S-S_{\mathrm{c}} \cong A_{+}\left|e_{2}\right|^{1-\alpha}$, а при $\Delta T<0$ они двузначны, и для двух фаз (жидкость-газ) имеем

$$
\begin{array}{ll}
\rho_{\text {Ж }}-\rho_{\mathrm{c}} \cong B\left|e_{2}\right|^{\beta}+b A_{-}\left|e_{2}\right|^{1-\alpha}, & \rho_{\Gamma}-\rho_{\mathrm{c}} \cong-B\left|e_{2}\right|^{\beta}+b A_{-}\left|e_{2}\right|^{1-\alpha}, \\
S_{\text {ж }}-S_{\mathrm{c}} \cong a B\left|e_{2}\right|^{\beta}+A_{-}\left|e_{2}\right|^{1-\alpha}, & S_{\Gamma}-S_{\mathrm{c}} \cong-a B\left|e_{2}\right|^{\beta}+A_{-}\left|e_{2}\right|^{1-\alpha},
\end{array}
$$

где $\beta=\left(d-\Delta_{1}\right) / \Delta_{2}, 1-\alpha=\left(d-\Delta_{2}\right) / \Delta_{2}(\alpha$ и $\beta$ - критические индексы теплоемкости и намагниченности в модели Изинга), $A_{ \pm}-$амплитудные множители $\partial_{2} p^{(\mathrm{s})}$ с двух сторон от $T_{\mathrm{c}}$, а $B$ - амплитудный множитель $\partial_{1} p^{(\mathrm{s})}$ при $\Delta T<0$. Вместо $e_{2}$ в формулах (7) можно использовать любую из величин $\Delta T, \Delta p, \Delta \mu$, что приведет лишь к изменению амплитуд (в частности, $\left.e_{2}=(1-a b) \Delta T\right)$. Если полностью пренебречь малой четной поправкой, для $\Delta T>0$ получим, что $\Delta \rho=0$ и $\Delta S=0$. Поэтому в реальном эксперименте с фиксированным количеством вешества линию $e_{1}=0$ выше температуры $T_{\mathrm{c}}$ можно приблизительно считать критической изохорой и (одновременно) критической адиабатой.

Обсудим в заключение вопрос об измеримости коэффициентов $a$ и $b$ в соотношениях (3). Если исходить из определения давления $p=p(T, \mu)$ с помощью стандартного соотношения $\ln Z=V p / k T$, где $Z$ - статсумма большого канонического ансамбля ( $V$ объем, $k$ - постоянная Больцмана) в термодинамическом пределе, то функция $p(T, \mu)$ и ее частные производные $S=\partial_{T} p$ и $\rho=\partial_{\mu} p$ теоретически вычислимы через статсумму $Z$. Однако выбор ансамбля - вопрос соглашения (подобно выбору калибровки потенциалов в электродинамике), поэтому следует различать понятия "вычислимость" и "измеримость". Величины $p$ и $\rho$ измеримы непосредственно, но для объемной плотности энтропии $S$ это не так. Общее термодинамическое определение энтропии [6] допускает произвол $\bar{S} \rightarrow \bar{S}+c$, где $\bar{S}=S / \rho$ - энтропия на одну частицу, $c-$ произвольная константа. При этом $F \rightarrow F-c N T$ для полной свободной энергии системы ( $N$ - число частиц) и $\mu \rightarrow \mu-c T$ для химического потенциала. Принимая в качестве исходных определений стандартные соотношения для большого канонического ансамбля [6], мы тем самым уже фиксируем некоторую конкретную нормировку энтропии и связанных с ней величин $F$ и $\mu$. Поэтому эти величины оказываются теоретически вычислимыми, не будучи непосредственно измеримыми (подобно потенциалам с фиксированной калибровкой в электродинамике).

Реально измеримыми являются лишь те величины, которые не зависят от отмеченного выше произвола в определении энтропии. Это, естественно, все величины, допускаюшие прямое экспериментальное измерение, в частности $p$ и $\rho$, теплоемкости $C_{v}$ и $C_{p}$ 
и сжимаемости (изотермическая и адиабатическая), но не $S$ и $\mu$. Произволу $\bar{S} \rightarrow \bar{S}+c$ в "энтропии на частицу" $\bar{S}=S / \rho$ соответствует произвол $S \rightarrow S+c \rho$ в ее объемной плотности, сохраняюшийся даже для разности $\Delta S=S-S_{\text {c. }}$ Однако для разности

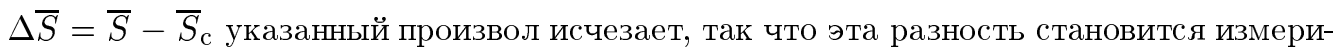
мой. В линейном приближении величина $\Delta \bar{S}$ имеет вид (2), поэтому из соотношений (7) можно получить аналогичные формулы для $\Delta \bar{S}$. Сравнивая амплитуды при однотипных вкладах в экспериментально измеримых величинах $\Delta \rho$ и $\Delta \bar{S}$, можно найти $a \rho_{\mathrm{c}}-S_{\mathrm{c}}$ и $b^{-1} \rho_{\mathrm{c}}-S_{\mathrm{c}}$, т.е. эти две комбинации измеримы (отметим, что по их разности можно найти отношение $(1-a b) / b$ и что комбинация $a \rho_{\mathrm{c}}-S_{\mathrm{c}}$ легко находится также по наклону кривой сосушествования вблизи ее окончания в плоскости $T, p)$.

Таким образом, по экспериментальным данным можно получить только две связи на три параметра $a, b$ и $S_{\mathrm{c}}$. Поэтому на феноменологическом уровне один из них ( $a$ или $S_{\mathrm{c}}$, но не $b$ ) можно было бы фиксировать произвольно, положив, например, $a=0$. Однако если считать исходной микромоделью большой канонический ансамбль, то поступить так нельзя, поскольку для него все три параметра $a, b, S_{\mathrm{c}}$ теоретически вычислимы.

\section{3. ПОЛНАЯ ДИНАМИЧЕСКАЯ ФЛУКТУАЦИОННАЯ МОДЕЛЬ}

Исходной фундаментальной микротеорией жидкости можно считать неравновесную статистическую физику системы взаимодействуюших классических частиш. В рамках этой теории корректно определены случайные поля $\widehat{F}(x)=\widehat{H}(x), \hat{p}_{i}(x), \hat{n}(x)$ - объемные плотности сохраняюшихся величин: энергии, импульса и числа частиц (для любых величин в динамике $x \equiv(t, \mathbf{x}))$. Их средние значения $\langle\ldots\rangle$ по равновесному распределению - известные термодинамические величины: $\langle\widehat{H}(x)\rangle=U$-внутренняя энергия единицы объема, $\langle\hat{n}(x)\rangle=\rho$ - плотность числа частиц, $\left\langle\hat{p}_{i}(x)\right\rangle=0$. Согласно общим принципам теории критического поведения Ландау [6] отклонения $\Delta \widehat{F}(x)=\widehat{F}(x)-$ $F_{\text {с }}$ случайных полей $\widehat{F}(x)$ от их равновесных средних значений в критической точке $\langle\widehat{F}(x)\rangle_{\mathrm{c}} \equiv F_{\mathrm{c}}$ можно считать "малыми" в критической области величинами, старшими степенями которых можно пренебрегать по сравнению с младшими (а в термодинамике с ними можно обрашаться как с дифференциалами). Тогда по исходным полям $\widehat{H}(x)-U_{\mathrm{c}} \equiv \Delta \widehat{U}(x)$ и $\hat{n}(x)-\rho_{\mathrm{c}} \equiv \Delta \hat{\rho}(x)$ с помошью термодинамического соотношения $\Delta U=T \Delta S+\mu \Delta \rho \cong T_{\mathrm{c}} \Delta S+\mu_{\mathrm{c}} \Delta \rho$ можно определить аналогичное случайное поле для объемной плотности энтропии $\Delta \widehat{S}(x)=T_{\mathrm{c}}^{-1}\left[\Delta \widehat{U}(x)-\mu_{\mathrm{c}} \Delta \hat{\rho}(x)\right]$, а вместо импульса $\hat{p}_{i}(x)$ можно ввести с той же точностью случайное поле скорости $\hat{v}_{i}(x)=\hat{p}_{i}(x) / M \rho_{\mathrm{c}}$, где $M$ - масса частицы. Переменная $v$ удобнее $p$ при обсуждении галилеевой инвариантности ("галилеево-ковариантным" аналогом $\partial_{t}$ является операция $\nabla_{t}=\partial_{t}+(v \partial)$ ). Хотя поле $v$ в отличие от импульса $p$ не является точно сохраняющейся величиной, из определений следует, что различием между ними в критической области можно пренебречь, т.е. все связанные с этим различием поправки должны быть "инфракрасно" (ИК)-несущественными величинами. Последующий строгий анализ, разумеется, подтвердит это утверждение.

Пять полей $\Delta \widehat{S}(x), \Delta \hat{\rho}(x)$ и $\hat{v}_{i}(x)$ образуют полный базис фигурируюших в динамике 
переменных

Равновесные средние $\langle\Delta \hat{\rho}(x)\rangle$ и $\langle\Delta \widehat{S}(x)\rangle$ - известные из статики величины [6]. Каждая из них содержит ведущий сингулярный вклад, пропорциональный $\partial_{1} p^{(\mathrm{s})}$ и менее сингулярный вклад $\sim \partial_{2} p^{(\mathrm{s})}$ с критическими размерностями $d-\Delta_{1}=\Delta_{\psi}$ и $d-\Delta_{2}=$ $\Delta\left[\psi^{2}\right]$, соответственно, где $\Delta_{F} \equiv \Delta[F]$ - критические размерности величин $F$ в простой $\psi^{4}$-модели $\left(\Delta_{1} \equiv \Delta_{h}, \Delta_{2} \equiv \Delta_{\tau}\right.$ для магнетика). Из соотношений (6) следует, что выделенными линейными комбинациями величин $\Delta \hat{\rho}(x)$ и $\Delta \widehat{S}(x)$ являются те, равновесные средние которых имеют определенные критические размерности $\partial_{1} p^{(\mathrm{s})}$ и $\partial_{2} p^{(\mathrm{s})}$, а именно,

$$
\begin{aligned}
\psi(x) & =\operatorname{const}[\Delta \rho(x)-b \Delta S(x)], \\
m(x) & =\operatorname{const}[\Delta S(x)-a \Delta \rho(x)],
\end{aligned}
$$

где $a$ и $b$ - коэффициенты из (3). В модели $\psi^{4}$ поле $\psi$ является параметром порядка, а поле $m$ представляется составным оператором $\psi^{2}$. Эквивалентная статическая задача с двумя независимыми полями $\psi$ и $m$ описывается известной “моделью C" [3] (подробнее см. ниже).

Выразив линейные комбинации (8), (9) через непосредственно измеримые величины $\Delta \rho$ и $\Delta \bar{S}$ с помошњю соотношений $(2)$ и $(6)$, получим $\psi \sim\left[\left(b^{-1} \rho_{\mathrm{c}}-S_{\mathrm{c}}\right) \Delta \rho-\rho_{\mathrm{c}}^{-2} \Delta \bar{S}\right]$ и $m \sim\left[\rho_{\mathrm{c}}^{2} \Delta \bar{S}-\left(a \rho_{\mathrm{c}}-S_{\mathrm{c}}\right) \Delta \rho\right]$. Отношение коэффициентов при $\Delta \bar{S}$ и $\Delta \rho$ в этих двух величинах выражается через измеримые комбинации параметров $a, b$ и $S_{\text {c }}$.

Именно комбинацию (8), имеющую определенную критическую размерность (в отличие от комбинации (2), являющейся смесью двух слагаемых с разной размерностью), следует считать основным полем параметра порядка Н-модели.

Уравнения гидродинамики идеальной жидкости для полей $\psi, m$ получаются из их определений (8), (9) и уравнений (1):

$$
\partial_{t} \psi=-\psi_{0} \partial_{i} v_{i}-\partial_{i}\left(v_{i} \psi\right), \quad \partial_{t} m=-m_{0} \partial_{i} v_{i}-\partial_{i}\left(v_{i} m\right),
$$

где $\psi_{0}$ и $m_{0}-$ некоторые константы. Добавив вклады затухания и шумов и интерпретируя слагаемые с $v$ как межмодовую связь, нетрудно достроить уравнения (10) до полной флуктуационной модели всех трех полей $\psi, m, v$ (см. ниже). В следующем разделе будет показано, что наличие “сцепления" между полем $\psi$ и продольной частью поля скорости $v_{\| \text {в }}(10)$ (которого не было бы для поля (2)) в действительности проблем не порождает, т.к. приводит лишь к некоторой перенормировке коэффициента Онзагера в стохастическом уравнении для $\psi$. Тем самым теряет силу основной аргумент в пользу выбора именно величины (2) в качестве поля параметра порядка. Поэтому предпочтительным становится выбор переменных (8), (9), поскольку именно эти комбинации имеют определенные критические размерности.

Опишем “полную флуктуационную модель" для трех полей $\psi, m, v$. Согласно общим принципам построения моделей критической динамики [3, 7] стохастические уравнения 
для произвольной системы полей $\varphi=\left\{\varphi_{\alpha}(x)\right\}$ с заданным статическим функционалом действия $S^{\mathrm{st}}(\varphi)$ должны иметь следуюший вид:

$$
\begin{aligned}
& \partial_{t} \varphi_{a}=\sum_{b}\left(\alpha_{a b}+\beta_{a b}\right) H_{\varphi_{b}}+\eta_{a} \\
& \left\langle\eta_{a}(x) \eta_{b}\left(x^{\prime}\right)\right\rangle \equiv D_{a b}\left(x, x^{\prime}\right)=2 \alpha_{a b} \delta\left(x-x^{\prime}\right),
\end{aligned}
$$

где $\eta=\left\{\eta_{a}(x)\right\}$ - набор случайных сил ("шумов") с гауссовым распределением, заданным матрицей корреляторов $(12), \alpha_{a b}$ - симметричная матрица коэффициентов Онзаге$\mathrm{pa}$, описываюших различные процессы диссипации, $\beta_{a b}$ - антисимметричная матрица коэффишиентов межмодовой связи. В (11) введено обозначение

$$
\left.H_{\varphi_{a}} \equiv \frac{\delta S^{\mathrm{st}}(\varphi)}{\delta \varphi_{a}(\mathbf{x})}\right|_{\varphi_{a} \mathbf{x} \rightarrow \varphi_{a}(x)}
$$

Термины “симметричность" для матрицы $\alpha$ и “антисимметричность" для матрицы $\beta$ при наличии в них операций дифференцирования нужно понимать как соотношения $\alpha_{b a}=\alpha_{a b}^{\mathrm{T}}, \beta_{b a}=\beta_{a b}^{\mathrm{T}}$ с обычным правилом транспонирования производных $\partial^{\mathrm{T}}=-\partial$.

Как известно, одновременные корреляционные функции полей $\varphi$ стохастической задачи (11), (12) совпадают со статическими корреляционными функциями тех же полей с функцией распределения $\exp \left(S^{\mathrm{st}}(\varphi)\right)$ (по смыслу $S^{\mathrm{st}}(\varphi)=-S_{0} / k T_{\mathrm{c}}$, где $S_{0}$ - функционал энергии Ландау). В этом и состоит свойство взаимной согласованности динамики и статики $[3,7]$.

Для нашей системы полей $\varphi=\{\psi, m, v\}$ в качестве $S^{\mathrm{st}}(\varphi)$ используется статический функционал действия С-модели для полей $\psi, m$ c простой добавкой приближенного выражения $M \rho_{\mathrm{c}} v^{2} / 2$ для кинетической энергии $[2,3,7]$ :

$$
S^{\mathrm{st}}(\varphi)=-\frac{(\partial \psi)^{2}}{2}-\frac{e_{2} \psi}{2}-\frac{g_{1} \psi^{4}}{24}+e_{1} \psi-\frac{m^{2}}{2}+\frac{g_{2} m \psi^{2}}{2}-\frac{a v^{2}}{2}
$$

где $e_{1,2}$ - параметры (3), $g_{1,2}$ - заряды, интегрирование по $d$-мерному аргументу х подразумевается. Параметр $a=M \rho_{\mathrm{c}} / k T_{\mathrm{c}}$ введен в (14) для того, чтобы сохранить обычньй физический смысл размерности поля скорости $v$. Поля $\psi$ и $m$ в функционале (14) имеют канонические размерности $d_{\psi}=d / 2-1$ и $d_{m}=d / 2$, что должно обеспечиваться выбором нормировочных множителей в (8), (9) в виде подходящих степеней параметра обрезания $\Lambda=r_{\min }^{-1}$, где $r_{\min }$ - минимальньй характерный размер для данной задачи межатомное расстояние.

В отсутствие диссипации и шумов все должно сводиться к уравнениям гидродинамики идеальной жидкости (10) для полей $\psi, m$. К ним нужно добавить известное [4] гидродинамическое уравнение для скорости $\partial_{t}\left(\bar{\rho} v_{i}\right)+\partial_{k} T_{i} k=0$, где $\bar{\rho}$ - массовая плотность среды, $T_{i k}$ - тензор натяжений, содержащий среди прочего "кинетический вклад" 
$\bar{\rho} v_{i} v_{k}$. В нашей задаче добавочные вклады $T_{i k}$, описываюшие взаимодействие скорости $v$ с полями $\psi$ и $m$, однозначно определяются изложенными вьше обшими принципами построения моделей критической динамики. Действительно, если интерпретировать вклады с $v$ в (10) как межмодовую связь полей $\psi$ и $m$ с $v$, то с учетом равенства $H_{v_{i}}=-a v_{i}$ для производной (13) функционала (14) из уравнений (10) находим коэффициенты межмодовой связи полей $\psi$ и $m$ с полем $v: \beta\left[\psi, v_{i}\right]=a^{-1} \partial_{i}\left[\left(\psi_{0}+\psi\right) \ldots\right]$, $\beta\left[m, v_{i}\right]=a^{-1} \partial_{i}\left[\left(m_{0}+m\right) \ldots\right]$. Отсюда по обшим правилам $\beta_{b a}=-\beta_{a b}^{\mathrm{T}}$ и $\partial^{\mathrm{T}}=-\partial$ определяются аналогичные коэффициенты межмодовой связи поля $v$ с полями $\psi$ и $m$ : $\beta\left[v_{i}, \psi\right]=a^{-1}\left(\psi_{0}+\psi\right) \partial_{i} \ldots, \beta\left[v_{i}, m\right]=a^{-1}\left(m_{0}+m\right) \partial_{i} \ldots$ В конечном итоге это приводит к следуюшему уравнению для скорости:

$$
\partial_{t} v_{i}+\left(v_{k} \partial_{k}\right) v_{i}=a^{-1}\left(\psi_{0}+\psi\right) \partial_{i} H_{\psi}+a^{-1}\left(m_{0}+m\right) \partial_{i} H_{m},
$$

где $H_{\psi}$ - соответствующие производные (13) функционала (14). Обеспечивающее галилееву инвариантность слагаемое $(v \partial) v$ в левой части уравнения (15) порождается кинетическим вкладом $\bar{\rho} v_{i} v_{k}$ в $T_{i k}$. Если бы поле скорости $v$ было чисто поперечным (несжимаемость), то вклад ( $v \partial) v$ (точнее, его “поперечная часть") был бы согласован со статикой (14), т.к. мог бы быть интерпретирован как межмодовое взаимодействие между компонентами $v$. Добавки от продольной части $v$ в слагаемое $(v \partial) v$ нарушают согласованность со статикой (14) и в этом смысле являются "плохими". В разделе 4 будет показано, что вклад ( $v \partial) v$ в действительности является ИК-несушественным, т.е. при анализе критической асимптотики может быть отброшен, и все связанные с ним проблемы исчезают. В принципе нетрудно изменить модель так, чтобы обеспечить полную согласованность со статикой. Для этого нужно изменить статическое действие (14) заменой приближенного выражения $\rho_{\mathrm{c}} v^{2}$ точным $\rho v^{2}$, изменив соответственно вклады межмодового взаимодействия в правой части уравнения (15) (что проще всего сделать в переменных $\Delta S, \Delta \rho)$, а вместо вклада $(v \partial) v$ в левой части (15) добавить в правую часть (15) межмодовое взаимодействие между компонентами $v$ с коэффициентами $\beta\left[v_{i}, v_{k}\right]=k T_{\mathrm{c}} \bar{\rho}^{-1}\left(\partial_{k} v_{i}-\partial_{i} v_{k}\right)$. При этом все отличия от простого уравнения (15) будут определяться ИК-несущественными поправками, которыми в критической области можно пренебречь. Поэтому в дальнейшем будем исходить из уравнений (10), (15).

$\mathrm{K}$ этим уравнениям нужно добавить скоррелированные между собой согласно соотношениям (11), (12) вклады диссипации и шумов. Разложив векторное поле скорости на продольную $\left(v_{\|}\right)$и поперечную $\left(v_{\perp}\right)$ составляющие, обозначим для краткости

$$
\left\{\psi, m, v_{\|}, v_{\perp}\right\} \equiv\left\{\varphi_{a}, \quad a=1,2,3,4\right\}
$$

и обсудим возможный обший вид $(4 \times 4)$-матришы коэффициентов Онзагера $\alpha_{a b}$. Он определяется с учетом следуюших соображений: 1) матриша $\alpha$ должна быть симметричной и положительно-определенной; 2) поперечное векторное поле $v_{\perp} \equiv \varphi_{4}$ не может смешиваться с прочими, поэтому $\left.\alpha_{4 a}=0 \forall a=1,2,3 ; 3\right)$ поскольку все наши поля порождаются сохраняюшимися величинами (см. начало раздела), все нетривиальные 
элементы $\alpha_{a b}$ должны содержать хотя бы один “множитель" $\partial[3,7]$. Отсюда с учетом индексной структуры и положительной определенности матрицы $\alpha$ следует, что все четыре диагональных элемента $\alpha_{a a}$ и недиагональный элемент $\alpha_{12}$ следует считать кратными оператору Лапласа $\partial^{2} \equiv \partial_{i} \partial_{i}$, а элементы $\alpha_{a 3}$ с $a=1,2$ - кратными оператору $\partial^{2} \partial_{i}$. Поясним последнее утверждение. Допустимый по индексной структуре вариант, когда $\alpha_{a 3} \sim \partial_{i}$ (т.е. $\alpha_{a 3} \sim p_{i}$ в импульсном представлении), противоречил бы при $p \rightarrow 0$ неравенству Коши-Буняковского $\alpha_{a 3}^{2} \leqslant \alpha_{a a} \alpha_{33} \sim p^{4}$, вытекающему из положительной определенности матрицы $\alpha$. Суммируя сказанное выше, можно ввести следующую параметризацию элементов матрицы $\alpha$ в обозначениях (16):

$$
\alpha_{a a}=-\lambda_{a a} \partial^{2} \quad \forall a ; \quad \alpha_{12}=-\lambda_{12} \partial^{2} ; \quad \alpha_{a 3}=-\lambda_{a 3} \partial^{2} \partial_{i}, \quad a=1,2,
$$

где все $\lambda$ - числовые коэффищиенты, при этом $\lambda_{a a}>0 \quad \forall a$.

Есть еше одно важное соображение относительно возможного вида матрицы $\alpha$. Если бы вместо полей $\psi, m$ в качестве переменных использовались исходные величины $\Delta S$ и $\Delta \rho$, то вклады от диссипации следовало бы ожидать в уравнениях для энтропии (теплопроводность) и скорости (вязкость), но их не должно быть в уравнении для плотности из-за отсутствия физического механизма “диссипации числа частиц". Это значит, что в таких переменных мы имели бы $\alpha_{\rho \rho}=0$, а поэтому и $\alpha_{\rho s}=\alpha_{\rho v}=0$ вследствие положительной определенности $\alpha$. Тогда отличными от нуля могли бы быть лишь элемент $\alpha_{s s}$, два (продольный и поперечный) элемента типа $\alpha_{v v}$ и один элемент типа $\alpha_{s v}$. Переход к переменным $\psi, m$ соответствует некоторому линейному преобразованию полей $\varphi \rightarrow L \varphi$, при котором $\eta \rightarrow L \eta, \alpha \rightarrow L \alpha L^{\mathrm{T}}$, что следует из соотношений $(11),(12)$. Поэтому из сказанного выше относительно структуры матрицы $\alpha$ в переменных $\Delta S, \Delta \rho, v$ вытекают вполне определенные связи для элементов новой матрицы $\alpha$ в переменных $\psi$, $m, v$. Легко проверить, что эти связи в обозначениях (16) имеют вид

$$
\frac{\alpha_{11}}{\alpha_{12}}=\frac{\alpha_{12}}{\alpha_{22}}=\frac{\alpha_{13}}{\alpha_{23}}=\frac{L_{11}}{L_{21}},
$$

где $L_{11}$ - коэффициент при $\Delta S$ в равенстве (8), а $L_{21}$ - в (9). Связи (18) эквивалентны аналогичным соотношениям на коэффициенты $\lambda$ в (17). Из (18), в частности, следует, что три элемента $\alpha_{11}, \alpha_{12}$ и $\alpha_{22}$ взаимно пропорциональны и могут быть равными нулю только одновременно.

Добавив к уравнениям (10), (15) вклады шумов и диссипации с коэффициентами Онзагера (17), согласно общим правилам (11), (12) получим следующую полную систему уравнений для полей $\varphi=\psi, m, v_{\|}, v_{\perp}$ :

$$
\begin{aligned}
& \partial_{t} \psi=-\lambda_{11} \partial^{2} H_{\psi}-\lambda_{12} \partial^{2} H_{m}+a \lambda_{13} \partial^{2}\left(\partial v_{\|}\right)-\partial(v \psi)-\psi_{0}\left(\partial v_{\|}\right)+\eta_{1}, \\
& \partial_{t} m=- \lambda_{22} \partial^{2} H_{m}-\lambda_{12} \partial^{2} H_{\psi}+a \lambda_{23} \partial^{2}\left(\partial v_{\|}\right)-m_{0}\left(\partial v_{\|}\right)-\partial(v m)+\eta_{2}, \\
& \partial_{t} v_{\|}=P^{\|}\left\{\lambda_{33} \partial^{2} v_{\|}-\lambda_{13} \partial\left(\partial^{2} H_{\psi}\right)-\lambda_{23} \partial\left(\partial^{2} H_{m}\right)-(v \partial) v+a^{-1} \psi \partial H_{\psi}+\right.\left.\quad+a^{-1} \psi_{0} \partial H_{\psi}+a^{-1} m \partial H_{m}+a^{-1} m_{0} \partial H_{m}\right\}+\eta_{3}, \\
& \quad \\
& \partial_{t} v_{\perp}=P^{\perp}\left\{a \lambda_{44} \partial^{2} v_{\perp}-(v \partial) v+a^{-1} \psi \partial H_{\psi}+a^{-1} m \partial H_{m}\right\}+\eta_{4},
\end{aligned}
$$


где $P^{\|}$и $P^{\perp}$ - продольный и поперечный проекторы $\left(P_{i k}^{\|}=\partial_{i} \partial_{k} / \partial^{2}, P_{i k}^{\perp}=\delta_{i k}-P_{i k}^{\|}\right)$, векторные индексы полей $v$ и символов $\partial$ для краткости опушены. Совокупность четырех уравнений (19) представляет собой “полную филуктуационную модель”, описывающую критическую динамику всех мягких мод в рассматриваемой задаче.

Отметим в заключение, что линии сосушествования фаз и ее прямолинейному продолжению - критической изохоре - соответствует выбор $e_{1}=0, e_{2} \sim \Delta T=T-T_{\mathrm{c}}$ в (14), а критический изобаре - выбор $e_{1} \sim e_{2} \sim \Delta T$ (для критической изотермы $\Delta T \rightarrow$ $\Delta p)$. Слагаемое $e_{1} \psi$ в $(14)$ порождает в $H_{\psi}$ константу $e_{1}$, вклад которой в уравнениях $(19)$ исчезает, т.к. $H_{\psi}$ входит в них только под знаком $\partial$. В такой ситуации для воспроизведения вклада $e_{1}$ необходимо искать решения уравнений $(19)$ для всех полей $\varphi(x)$ в виде $\varphi^{\prime}+\varphi^{\prime \prime}(x)$, где $\varphi^{\prime \prime}(x)$ - убывающие при $|\mathbf{x}| \rightarrow \infty$ для всех $t$ “флуктуационные составляющие", а $\varphi^{\prime}$ - константы, определяемые из условия минимума энергии $H_{\varphi}=0$ для всех $\varphi$ на не зависящих от $x$ полях $\varphi$.

\section{4. ИСКЛЮЧЕНИЕ ЗВУКОВЫХ МОД}

Мы привели громоздкие общие уравнения для полей (19). Докажем теперь, что многие вклады в этих уравнениях являются ИК-несушественными. Поэтому при анализе ведущих критических сингулярностей они могут быть отброшены. В конечном счете данная процедура упрощения модели (19) приведет к полному исключению звуковых мод $m, v_{\|}$и к обычной формулировке Н-модели, но только с полем параметра порядка (8) вместо обычного поля (2).

Переходим к доказательству этого утверждения. Согласно общим принципам построения фолуктуационных моделей теории критического поведения [6] судить об относительной ИК-существенности различных слагаемых в уравнениях (19) можно просто по размерности входящих в них числовых коэффициентов. Речь идет лишь о таких коэффициентах, которые остаются конечными в исследуемой критической асимптотике. Важно также, чтобы сами поля соответствовали величинам с определенными размерностями, а не смесям таких величин, что и согласуется с выбором полей (8), (9) в качестве динамических переменных.

В динамических задачах аналогом обычной (импульсной) размерности статических моделей является "суммарная каноническая размерность"

$$
d_{F}=d_{F}^{p}+d_{\omega} d_{F}^{\omega},
$$

где $d_{F}^{p}$ и $d_{F}^{\omega}$ - соответственно импульсная и частотная канонические размерности рассматриваемой величины $F, d_{\omega}$ - относительная размерность частоты. Последняя определяется по соотношению между $\partial_{t}$ и $\partial$ в линейной части стохастических уравнений при $T=T_{\mathrm{c}}$, т.е. непосредственно в критической точке. В нашем случае из уравне-

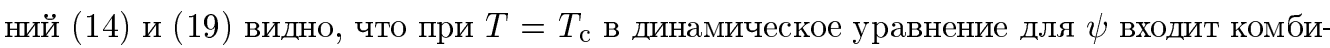
нация $\partial_{t}+$ const $\cdot \partial^{4}$, а в уравнения для полей $m$ и $v$ входит $\partial_{t}+$ const $\cdot \partial^{2}$. Поэтому выбор величины $d_{\omega}$ в данном случае неоднозначен (либо $\omega \sim p^{4}$, либо $\omega \sim p^{2}$ ) и в такой ситуации должен пониматься просто как один из элементов точной постановки асимптотической задачи. 
Общепринятым для Н-модели является выбор $\omega \sim p^{4} \quad\left(d_{\omega}=4\right)$, соответствуюший полноценной динамике для поля параметра порядка $\psi$. Он и принимается в дальнейшем, как и выбор $d=4-2 \epsilon$ размерности пространства, поскольку оба статических взаимодействия в (14), как и межмодовое взаимодействие $\psi$ с $v$ в уравнениях (15), логарифмичны при $d=4$. Нужные для дальнейшего сведения о суммарных размерностях (20) входящих в систему (19) величин при $d_{\omega}=4, d=4$ приведены в следующей таблице.

\begin{tabular}{|c|c|c|c|c|c|c|c|c|c|c|}
\hline$F$ & $\psi, \psi_{0}$ & $m, m_{0}$ & $v$ & $H_{\psi}$ & $H_{m}$ & $a$ & $\lambda_{11}$ & $\lambda_{12}, \lambda_{13}$ & $\lambda_{22}, \lambda_{23}$ & $\lambda_{33}, \lambda_{44}$ \\
\hline$d_{F}$ & 1 & 2 & 3 & 3 & 2 & -2 & 0 & 1 & 2 & 4 \\
\hline
\end{tabular}

Размерности $d_{F}$ полей $\psi, m$ находятся по виду функционала $(14)$, размерность скорости - непосредственно из ее определения (условно $v\left(\right.$ см/c) $\omega / p \sim p^{3}$ ), по ней из (14) находится $d_{a}$. Размерности шумов $\eta_{a}$ в уравнениях (19) совпадают с размерностями производных $\partial_{t} \varphi_{a}$, по ним из соотношений (12) находятся размерности всех $\lambda_{a b}$ в $(17)$.

Из таблицы видно, что числовые коэффициенты при различных слагаемых (19) имеют разные суммарные размерности, поэтому некоторые из них заведомо могут быть отброшены как ИК-несущественные. По общим правилам в каждом из уравнений (19) самыми главными являются те вклады, числовые коэффициенты при которых имеют максимальные суммарные размерности. При такой оценке вклады шумов $\eta_{a}$ мы считаем представленными в виде

$$
\eta_{a}=\lambda_{a a}^{1 / 2} \eta_{a}^{\prime}
$$

рассматривая множители $\lambda_{a a}^{1 / 2}$ как числовые коэффициенты на равных правах со всеми прочими.

Для краткости будем использовать термины “порядок вклада" и “его индекс", понимая последний как суммарную размерность числового коэффициента в этом вкладе. Индексы всех вкладов в уравнениях (19) легко находятся из таблицы. В частности, из (21) следует, что индексы шумов $\eta_{1}, \eta_{2}, \eta_{3}, \eta_{4}$ в (19) равны соответственно $0,1,2,2$.

Поскольку нас интересуют только ведушие сингулярности в критической области, из сказанного выше на первый взгляд следует, что в каждом из уравнений (19) нужно оставить лишь главные вклады с максимальным индексом $(+1$ в уравнении для $\psi,+2$ в уравнениях для $m$ и $v_{\perp},+4$ в уравнении для $\left.v_{\|}\right)$. В таком приближении уравнения для $v_{\|}$и $m$ сводятся к простым соотношениям

$$
\partial H_{m}=0, \quad \partial v_{\|}=0
$$

при учете которых уравнение для $\psi$ становится тождеством $(0=0)$. Содержательным остается лишь уравнение для $v_{\perp}$.

Однако в действительности таким приближением ограничиваться в данном случае нельзя, т.к. "малые” величины (22) входят в оба вклада $-\lambda_{12} \partial^{2} H_{m}$ и $-\psi_{0}\left(\partial v_{\|}\right)$с максимальным индексом +1 в уравнении для $\psi$. Поэтому сейчас важны и поправки к ним с относительным индексом -1 , которые после умножения на коэффициенты $\lambda_{12}$ и $\psi_{0} \mathrm{c}$ 
размерностью +1 дадут в уравнении для $\psi$ добавки с нулевым индексом, т.е. вклады того же порядка, что и у большинства других слагаемых этого уравнения.

Таким образом, из-за “аномальной малости” величин (22) ведушим порядком в уравнении для $\psi$ являются все вклады с нулевым $($ а не равным +1$)$ индексом, и для их определения нужно учитывать не только самые главные (с максимальным индексом) слагаемые, но также и все "первые поправки", т.е. все вклады с индексом на единицу меньше максимального. Сразу отметим, что поправки следуюшего порядка с уменшшением индекса на 2 и более заведомо ИК-несушественны и могут быть отброшены. К их числу относятся все вклады с $\lambda_{13}$ и $\lambda_{23}$, а также вклады $(v \partial) v$ и $\partial_{t} v$ во всех уравнениях. Поэтому проблема нарушения согласованности со статикой из-за вкладов с $v_{\|}$в $(v \partial) v$ фактически снимается.

Учитывая в уравнениях для $v_{\|}$и $m$ все первые поправки, получаем

$$
\begin{aligned}
& 0=\psi_{0} \partial H_{\psi}+m_{0} \partial H_{m} \\
& 0=-\lambda_{22} \partial^{2} H_{m}-\lambda_{12} \partial^{2} H_{\psi}-m_{0}\left(\partial v_{\|}\right)+\eta_{2} .
\end{aligned}
$$

Найдя отсюда “малые величины” $\partial H_{m}, \partial v_{\|}$, подставив их в уравнения $(19)$ для $\psi$ и $v_{\|}$, а затем отбросив в правых частях этих уравнений все ИК-несушественные поправочные вклады (с отрицательным индексом в уравнении для $\psi$ и с индексом меньше двух в уравнении для $\left.v_{\|}\right)$, получим следующую систему:

$$
\begin{aligned}
& \partial_{t} \psi=-\lambda_{11}^{\prime} \partial^{2} H_{\psi}-\partial(v \psi)+\eta_{1}^{\prime}, \\
& \partial_{t} v_{\|}=P^{\perp}\left\{a \lambda_{44} \partial^{2} v_{\perp}+a^{-1} \psi \partial H_{\psi}\right\}+\eta_{4},
\end{aligned}
$$

в которой

$$
\eta_{1}^{\prime}=\eta_{1}-\alpha \eta_{2}, \quad \lambda_{11}^{\prime}=\lambda_{11}-2 \alpha \lambda_{12}+\alpha^{2} \lambda_{22}, \quad \alpha \equiv \psi_{0} / m_{0}
$$

Для сохранения традиционной формы записи мы оставили в уравнении (25) и вклад $\partial_{t} v_{\perp}$, хотя он является ИК-несушественным, т.к. имеет индекс 0, а все слагаемые в правой части $(25)-$ индекс +2 . Если умножить обе части $(25)$ на коэффициент $a$ с $d_{a}=$ -2 (см. таблищу), все вклады в правой части (25) будут иметь индекс 0, как и все слагаемые уравнения $(24)$, а вклад $a \partial_{t} v_{\perp}$ в левой части уравнения для $v_{\perp}$ будет явно ИК-несущественной поправкой с индексом - 2, которой при анализе ведущих ИК-сингулярностей можно пренебречь. Равенство нулю индексов всех вкладов главного порядка в конечных уравнениях - основной признак "хорошей” (логарифмической) теории, т.к. все добавки с отрицательными индексами ИК-несушественны (важно, чтобы не было вкладов с положительными индексами).

В записи (24), (25) поля $m$ и $v_{\|}$еше не устранены полностью, поскольку $m$ входит в производную $H_{\psi}(13)$ функционала $(14)$, а $v_{\|}$входит в $\partial(v \psi)$. Для полного исключения полей $m$ и $v_{\|}$нужно воспользоваться уравнениями связи $(22)$, в данном случае уже без поправок из (23), поскольку они будут порождать в уравнениях $(24),(25)$ лишь заведомо ИК-несушественные вклады. Из (22) следует, что $H_{m}=$ const, $v_{\|}=$const и обе 
эти константы должны быть равны нулю даже при наличии у полей постоянных (не зависящих от $x$ ) составляюших по условиям минимума энергии (см. замечание в конце раздела 3 ). Таким образом, с учетом явного вида производной $H_{m}$ функционала (14) получаем

$$
H_{m}=-m+\frac{g_{2} \psi^{2}}{2}=0, \quad v_{\|}=0
$$

Отсюда следует, что поле $v$ в уравнениях (24), (25) можно считать чисто поперечным, а поле $m$ можно выразить явно через $\psi$ с помошью первого соотношения (27). Это позволяет исключить $m$ из производной $H_{\psi}$ в $(24),(25)$. Легко проверить, что данная процедура эквивалентна исключению $m$ с помощью соотношения (27) непосредственно в самом функционале (14), который перейдет тогда в статическое действие простой $\psi^{4}$-модели (плюс вклад $v^{2}$ с $v \equiv v_{\perp}$ согласно $(27)$ )

$$
S^{\mathrm{st}}(\psi, v)=-\frac{(\partial \psi)^{2}}{2}-\frac{e_{2} \psi^{2}}{2}-\frac{g^{\prime} \psi^{4}}{24}+e_{1} \psi-\frac{a v^{2}}{2}
$$

с зарядом $g^{\prime} \equiv g_{1}-3 g_{2}^{2}$.

Вытекающая из (27) поперечность поля $v$ в $(24),(25)$ позволяет сделать в уравнении $(24)$ замену $\partial(v \psi) \rightarrow(v \partial) \psi$, и тогда этот вклад вместе с $\partial_{t} \psi$ комбинируется в галилеево-ковариантную производную $\nabla_{t} \psi=\left[\partial_{t}+(v \partial)\right] \psi$, чем обеспечивается галилеева инвариантность уравнения для $\psi$. Аналогичное свойство уравнения для $v_{\perp}$ будет иметь место при одновременном отбрасывании (или сохранении) ИК-несушественных вкладов $\partial_{t} v$ и $(v \partial) v$.

После исключения полей $m$ и $v_{\|}$из $(24),(25)$ получаем соотношения

$$
\begin{aligned}
& \partial_{t} \psi=-\lambda_{11}^{\prime} \partial^{2} H_{\psi}-(v \partial) \psi+\eta_{1}^{\prime}, \\
& a \partial_{t} v_{\perp}=P^{\perp}\left\{a^{2} \lambda_{44} \partial^{2} v_{\perp}+\psi \partial H_{\psi}\right\}+a \eta_{4},
\end{aligned}
$$

в которых $H_{\psi}$-производная (13) статического действия (28). С точностью до обозначений соотношения (29), (30) совпадают с обычными уравнениями Н-модели [2, 3] (только с иным физическим смыслом поля $\psi$ ). По общему правилу (см., например, [7]) стохастическая задача (29), (30) эквивалентна квантово-полевой модели с удвоенным числом полей $\Phi=\psi, v, \psi^{\prime}, v^{\prime}$ и следуюшим динамическим функционалом действия:

$$
\begin{aligned}
S(\Phi)= & -\lambda_{11}^{\prime} \psi^{\prime} \partial^{2} \psi^{\prime}+\psi^{\prime}\left[-\partial_{t} \psi-\lambda_{11}^{\prime} \partial^{2} H_{\psi}-(v \partial) \psi\right]- \\
& -a^{2} \lambda_{44} v^{\prime} \partial^{2} v^{\prime}+v^{\prime}\left[-a \partial_{t} v+a^{2} \lambda_{44} \partial^{2} v+\psi \partial H_{\psi}\right] .
\end{aligned}
$$

Векторные поля $v, v^{\prime}$ в (31) считаются поперечными, поэтому проектор $P^{\perp}$ перед вкладом $\psi \partial H_{\psi}$ можно опустить.

Обсудим теперь коэффициенты Онзагера в уравнениях $(24),(25)$ и (29), (30). По своему смыслу комбинация $a \lambda_{44}$ в $(25)$ - кинематический коэффициент вязкости, а $\lambda_{11}^{\prime}$ в (24) - коэффициент теплопроводности (для неренормированной задачи все эти параметры являются затравочными). Отметим, что произошедшая при исключении полей $m$ и $v_{\|}$ 
перенормировка $\lambda_{11} \rightarrow \lambda_{11}^{\prime}(26)$ коэффициента Онзагера в уравнении для $\psi$ точно соответствует по правилам (11), (12) перенормировке $\eta_{1} \rightarrow \eta_{1}^{\prime}$ соответствуюшего случайного шума. При этом новый шум $\eta_{1}^{\prime}$ оказывается точно таким же, как если бы мы с самого начала писали релаксационное уравнение для “энтропии на частицу” (2), а не для нашего поля (8). Это очевидно из выражения (26) для $\eta_{1}^{\prime}$ : формально такой шум можно было бы получить, если бы мы умножили обе части уравнения (19) для поля $m$ на коэффициент $\alpha=\psi_{0} / m_{0}$ и затем выгли его из уравнения $(19)$ для $\psi$. Тогда вклад $\psi_{0}\left(\partial v_{\|}\right)$в этом уравнении сократился бы, а под знаком $\partial_{t}$ появилась комбинация $\psi-\alpha m$, кратная, очевидно, величине (2), т.к. относительный коэффициент между $\Delta S$ и $\Delta \rho$ в (2) однозначно определяется именно требованием взаимного сокрашения $\partial v_{\|}$в линеаризованных уравнениях гидродинамики. Это доказывает, что определенный соотношением (26) параметр $\lambda_{11}^{\prime}$ в (24) и есть тот самый коэффициент теплопроводности, который используется обычно [1-3], когда релаксационные уравнения пишутся непосредственно для “энтропии на частицу” (или “энтропии на единицу массы”) (2).

Добавим в заключение, что вместо уравнений (19) для полей $\psi \equiv \varphi_{1}$ и $m \equiv \varphi_{2}$ можно было бы, конечно, написать аналогичные уравнения для любой другой пары переменных $\varphi^{\prime}=\varphi_{1}^{\prime}, \varphi_{2}^{\prime}$, связанных с $\varphi=\varphi_{1}, \varphi_{2}$ произвольным линейным преобразованием $\varphi^{\prime}=L \varphi$, и это привело бы к соответствующему преобразованию шумов и коэффициентов Онзагера: $\eta \rightarrow \eta^{\prime}=L \eta, \alpha \rightarrow \alpha^{\prime}=L \alpha L^{\mathrm{T}}$. Полезно отметить, что входящая в уравнение (15) для $\partial_{t} v$ комбинация $\sum_{\alpha}\left(\varphi_{0 \alpha}+\varphi_{\alpha}\right) \partial H_{\varphi_{\alpha}} \equiv\left(\varphi_{0}+\varphi\right) \partial H_{\varphi}$ является инвариантом любых линейных преобразований: $\left(\varphi_{0}+\varphi\right) \partial H_{\varphi}=\left(\varphi_{0}^{\prime}+\varphi^{\prime}\right) \partial H_{\varphi^{\prime}} \forall \varphi^{\prime}=L \varphi$. По физическим соображениям выделенной парой переменных является $\varphi_{1}^{\prime}=\Delta \bar{S}(2)$ и $\varphi_{2}^{\prime}=\Delta \rho$, т.к. для первой из них в линейном приближении нет сцепления с $\partial v_{\|}$(т.е. $\left.\varphi_{01}^{\prime}=0\right)$, а для второй нет диссипации и соответствуюшего шума (т.е. $\eta_{2}^{\prime}=0$ ). Для таких переменных в уравнении для $\partial_{t} \varphi_{1}^{\prime}$ не будет вкладов типа $\operatorname{const}\left(\partial v_{\|}\right)$и $\alpha_{12}^{\prime} H_{2}^{\prime}$, т.е. тех самых двух слагаемых, которые порождали некоторые проблемы (впрочем, легко решаемые) в аналогичном уравнении (19) для $\psi$. Однако для последуюшего “анализа по размерности" ИК-существенности различных вкладов в уравнениях, необходимого для их упрошения, все равно придется вернуться к "правильным" (в смысле размерностей) переменным $\varphi=\psi, m$. В конечном счете после упрощений мы все равно придем к уравнению (24) для нашего поля $\psi$ независимо от выбора пары исходных переменных $\varphi^{\prime}$, а второе уравнение в ведушем (по индексу) порядке сведется к связи $\partial v_{\|}=0$.

\section{5. УРАВНЕНИЯ ДЛЯ ЗВУКОВЫХ ВОЛН}

Полная модель (19) является естественной основой для вывода уравнений, описывающих распространение звука в окрестности критической точки. Взаимодействие звуковой волны с критическими флуктуациями ведет к появлению критических сингулярностей в скорости звука и в коэффициенте затухания. Экспериментально эти эффекты наблюдаются обычно в ультразвуковом диапазоне [8].

В литературе предлагались разные способы вывода уравнений для звуковых волн и соответствуюшего закона дисперсии, основанные на разных вариантах "полной фллук- 
туационной модели" с разным выбором ее динамических переменных [9-11]. Здесь мы приведем еше один вариант вывода звуковых уравнений, на наш взгляд, более четкий и прозрачньй, причем (что важно) оперирующий с “правильными” динамическими переменными $\psi, m$. Для определенности будем полагать в (14) $e_{1}=0, e_{2}=T-T_{\mathrm{c}} \equiv \tau$ с точностью до нормировки (критическая изохора), что соответствует постановке эксперимента (заданное количество вешества в заданном объеме).

Приступая к самому выводу, для конкретизации задачи допустим, что к случайным шумам $\eta_{2,3}$ в уравнениях (19) добавлены сколь угодно слабые по интенсивности внешние (т.е. фиксированные, а не случайные) гармонические (т.е. кратные $\exp (-i \omega t))$ с заданной произвольной звуковой частотой $\omega$ ) источники $\delta \eta_{2,3}$. Тогда в решениях для полей $m$ и $v_{\|}$появятся малые гармонические добавки $\theta=\left\{\delta m, \delta v_{\|}\right\}$, которые в дальнейшем будем называть "волновыми" (или "звуковыми”) составляюшими. Взаимодействие всех полей в (19) приведет, естественно, к появлению аналогичных звуковых добавок и у полей $\psi$ и $v_{\perp}$, но нас они будут интересовать лишь постольку, поскольку они будут влиять на решения для $\theta=\left\{\delta m, \delta v_{\|}\right\}$.

Итак, рассмотрим уравнения (19) с добавками $\delta \eta_{2,3}$ и в качестве первого шага, как и раньше, выполним процедуру исключения в уравнении для $\psi$ вкладов $\lambda_{12} \partial^{2} H_{m}$ и $\psi_{0}\left(\partial v_{\|}\right)$с индексом +1 . Для этого перепишем точные уравнения (19) для $m$ и $v_{\|}$с добавками $\delta \eta_{2,3}$ в виде $m_{0}\left(\partial v_{\|}\right)=$"все прочее", $a^{-1} m_{0} \partial H_{m}=$ "все прочее", выразим отсюда через "все прочее" величины $\partial v_{\|}$и $\partial H_{m}$, подставим их в уравнения (19) для $\psi$ и $v_{\perp}$, а затем отбросим в их правых частях все ИК-несушественные по индексу вклады. Отметим, что при этом будут исключены и все кратные $\delta \eta_{2,3}$ добавки (т.е. эти добавки имеют смысл "волновых поправок" в ИК-несушественных вкладах, поэтому и отбрасываются вместе с последними). В итоге для полей $\psi$ и $v_{\perp}$ мы получим прежние уравнения $(24),(25)$. Следует подчеркнуть, что это еше не означает полную потерю вкладов волновых поправок $\theta=\left\{\delta m, \delta v_{\|}\right\}$в уравнениях для $\psi$ и $v_{\perp}$, т.к. в уравнениях $(24),(25)$ все еше остаются поля $m$ и $v_{\|}$, содержашие эти поправки.

Таким образом, четыре уравнения (19) заменяются парой уравнений (24), (25) для полей $\psi, v_{\perp}$ и парой уравнений (19) для полей $m$ и $v_{\|}$с добавками звуковых источников $\eta_{2,3}$. Такая замена выгодна тем, что в уравнении (24) уже нет вкладов с индексом +1 , поэтому при исключении из уравнений $(24),(25)$ полей $m$ и $v_{\|}$можно ограничиться ведушим (без поправок) приближением для этих полей. Это значит, что при расчете полей $m$ и $v_{\|}$с помошью соответствуюших уравнений (19) в них уже можно пренебрегать всеми ИК-несушественными поправками, ограничиваясь ведушими вкладами.

В отсутствие волновых добавок уравнения (19) для $m$ и $v_{\|}$в ведушем (по индексу) приближении сводились к равенствам $0=-\lambda_{22} \partial^{2} H_{m}-m_{0}\left(\partial v_{\|}\right), 0=a^{-1} m_{0} \partial H_{m}$, что приводило к связям (22) и их следствиям (27), из которых получалось, что $v_{\|}=0, m=$ $g_{2} \psi^{2} / 2 \equiv \bar{m}$. При введении источников $\delta \eta_{2,3}$ появляются соответствуюшие волновые добавки $\delta v_{\|}=v_{\|}, \delta m=m-\bar{m}$, при этом $H_{m}=-\delta m$ согласно первому равенству (27).

Для вычисления волновых добавок в уравнениях (19) для $m$ и $v_{\|}$, кроме приведенных выше главных (по индексу) слагаемых, следует также сохранить и вклады $\partial_{t} m, \partial_{t} v_{\|}$в левых частях, которые, очевидно, совершенно необходимы для описания звуковых волн. 
Пренебрегая всеми прочими вкладами в (19) как ИК-несущественными, получаем следующую систему звуковых уравнений:

$$
\partial_{t} m=-\lambda_{22} \partial^{2} H_{m}-m_{0}\left(\partial v_{\|}\right)+\delta \eta_{2}, \quad \partial_{t} v_{\|}=a^{-1} m_{0} \partial H_{m}+\delta \eta_{3},
$$

в которых $m=\bar{m}+\delta m, \bar{m}=g_{2} \psi^{2} / 2, H_{m}=-\delta m$.

Слагаемое с $\lambda_{22}$ в системе (32) определяет затухание звуковой волны за счет эффектов теплопроводности. В дальнейшем мы будем им пренебрегать, т.к. эти эффекты в критической области несущественны по сравнению с затуханием, порождаемым взаимодействием волны с критическими флуктуациями (аналогичный вклад в затухание от продольной вязкости в системе (32) уже отброшен). Опустив в $(32)$ вклад с $\lambda_{22}$ и подставив явные выражения для $m$ и $H_{m}$, получим

$$
\partial_{t}\left[\delta m+\frac{g_{2} \psi^{2}}{2}\right]+m_{0}\left(\partial v_{\|}\right)=\partial \eta_{2}, \quad \partial_{t} v_{\|}+a^{-1} m_{0} \partial \delta m=\delta \eta_{3} .
$$

Взаимодействие звуковой волны с критическими флуктуациями определяется вкладом, пропорциональным $\psi^{2}$. Без него соотношения (33) являются простой системой линейных неоднородных уравнений $L_{0} \theta=J$ для полей $\theta=\delta m, v_{\|}$и источников $J=$ $\delta \eta_{2}, \delta \eta_{3}$ с $(2 \times 2)$-матричной дифференциальной операцией $L_{0}$. Условие $\operatorname{det} L_{0}=0$ приводит к стандартному закону дисперсии

$$
\omega=c_{0} k, \quad c_{0}^{-2}=a m_{0}^{-2}
$$

константа $c_{0}-$ скорость звука в данном приближении.

Теперь нужно учесть влияние возмушения, пропорционального $\psi^{2}$, в уравнениях (33). При оценке этого влияния необходимо иметь в виду очень важное качественное различие между звуковыми $\left(\theta \equiv \delta m, v_{\|}\right)$и флуктуационными $\left(\varphi \equiv \psi, v_{\perp}\right)$ полями. Для последних характерным размером при $\tau \equiv\left|T-T_{\mathrm{c}}\right| \rightarrow 0$ (на критической изотерме $\Delta T \rightarrow$ $\Delta p$ ) является корреляционная длина $\xi \sim \tau^{-\nu}$, характерным импульсом (волновым вектором) - величина $k_{\text {фл }}=\xi^{-1} \sim \tau^{\nu}$, характерной частотой-величина $\omega_{\text {фл }} \sim k_{\text {фл }}^{z} \sim \tau^{z \nu}$, где $z \equiv \Delta_{\omega}-$ критическая размерность частоты в Н-модели. Качественные оценки естественно делать просто по каноническим размерностям, т.е. без учета аномалий. Тогда $\nu=1 / 2, z=4$ и при $\tau \rightarrow 0$ получаем, что $k_{\text {фл }} \sim \tau^{1 / 2}$ и $\omega_{\text {фл }} \sim k_{\text {фл }}^{4} \sim \tau^{2}$. Для зву-

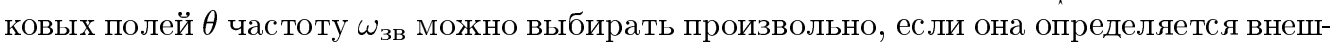
ним источником. С точки зрения эксперимента интерес представляет широкая область частот, в которой $\omega_{3 в}$ может быть как много меньше, так и много больше характерной флуктуационной частоты $\omega_{\text {фл }}$. Поэтому при качественной оценке их можно считать величинами одного порядка: $\omega_{\text {зв }} \sim \omega_{\text {фл }} \sim \tau^{2}$. Отсюда для характерных волновых векторов звуковых полей получается $k_{\text {зв }}=c_{0}^{-1} \omega_{\text {зв }} \sim \tau^{2}$, что приводит к неравенству $k_{\text {зв }} \sim \tau^{2} \ll k_{\text {фл }} \sim \tau^{1 / 2}$ при $\tau \rightarrow 0$. По смыслу $k_{\text {зв }}=\lambda_{\text {зв }}^{-1}-$ обратная длина звуковой волны, а $k_{\text {фл }}=\xi^{-1}$ - обратная корреляционная длина, так что приведенное выше неравенство эквивалентно неравенству $\lambda_{\text {зв }} \gg \xi$. Это означает, что длина звуковой волны 
много больше характерного размера флуктуаций $\xi$. Поэтому при оценке обратного влияния звуковых полей $\theta$ на флуктуационные поля Н-модели поля $\theta$ можно рассматривать как "почти пространственно-однородные" (хотя скорость их изменения сопоставима с аналогичной величиной для флуктуационных полей). Неравенство $\lambda_{3 в} \gg \xi$ выполняется во всей достижимой экспериментально области значений $\omega_{3 в}$ и $\tau$, причем даже тогда,

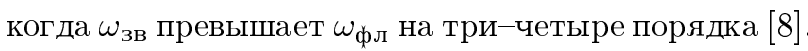

Вернемся к уравнениям (33). Входящую в них величину $\psi^{2}$ нужно рассматривать как соответствующее случайное поле Н-модели, "слегка возмущенной" влиянием на нее звуковых полей. Это возмушение слабое (т.к. звуковые поля определяются источниками в (33) и поэтому могут считаться сколь угодно мальми), но полностью пренебречь им нельзя, т.к. пропорциональные звуковым полям $\theta$ поправки $\delta \psi^{2}$ в случайной величине $\psi^{2}$ сопоставимы с прочими вкладами в (33). Для любой построенной из полей Н-модели случайной величины $F$ эти поправки определяются соотношением $\delta F=F \delta S$, где $\delta S$ - линейная по звуковым полям $\theta$ поправка к динамическому действию (31) Н-модели. Действительно, функция распределения для полей $\phi$ возмушенной Н-модели определяется в функциональных интегралах по $\phi$ весовым множителем $\exp [S(\phi)+\delta S(\phi)]$, сводяшимся к $[1+\delta S(\phi)] \exp S(\phi)$ в линейном приближении по возмущению. Отсюда, в частности, следует, что для случайной величины $\psi^{2}$ в $(33)$

$$
\left.\psi^{2}(x)\right|_{\text {точн }} \cong \psi^{2}(x)+\psi^{2}(x) \delta S(\phi) .
$$

Все поля в правой части соотношения (35) имеют смысл соответствуюших случайных величин простой Н-модели без поправок, поскольку их распределение определяется (см. выше) весовым множителем $\exp S(\phi)$.

При подстановке (35) в (33) не зависящее от звуковых полей $\theta$ первое слагаемое в правой части (35) будет порождать лишь некоторую "флуктуационную" (и при этом не гармоническую) добавку к источнику $\delta \eta_{2}$, которую в дальнейшем будем опускать, т.к. она не оказывает влияния на интересуюший нас закон дисперсии звуковых волн. В этом смысле важна лишш линейная по полям $\theta$ поправка $\psi^{2} \delta S(\phi)$ в (35). Величину $\delta S(\phi)$ нетрудно найти по поправкам $\sim \theta$ в уравнениях $(24),(25)$. Они порождаются, во-первых, вкладом $v_{\|}$в $\partial(v \psi)$, во-вторых, вкладом $g_{2} \psi m$ в производную $H_{\psi}$ функционала (14), что дает добавку $\delta H_{\psi}=g_{2} \psi \delta m$. Все эти поправки в уравнениях (24), (25) соответствуют добавке

$$
\delta S(\phi)=\psi^{\prime}\left[-\lambda_{11} g_{2} \partial^{2}(\psi \delta m)-\partial\left(v_{\|} \psi\right)\right]+v_{\perp}^{\prime}\left[g_{2} \psi \partial(\psi \delta m)\right]
$$

к динамическому действию (31) Н-модели.

Мы привели полное выражение для $\delta S(\phi)$, но фактически в нем сушественно только первое слагаемое. Действительно, нас интересует лишь регулярная гармоническая составляюшая звуковых полей $\theta$, которая порождается вкладом гармонических внешних источников в уравнениях (33). Порождаемая вкладом, пропорциональным $\psi^{2}$, невозмущенной Н-модели аддитивная "флуктуационная добавка" в $\theta$ нас не интересует, и мы условились ее отбрасывать. Считая это сделанным, под $v_{\|}$и $\delta m$ в (36) можно понимать лишь малые гармонические составляюшие этих полей. В силу неравенства $\lambda_{\text {зв }} \gg \xi$ эти 
величины в рамках Н-модели можно рассматривать как "почти пространственно однородные", поэтому можно пренебрегать их градиентами. В таком приближении в правой части равенства (36) исчезает последний вклад (вследствие поперечности $v_{\perp}$ ), а в первом выражение $\partial^{2}(\psi \delta m)$ можно заменить на $\left(\partial^{2} \psi\right)(\delta m)$. Эта добавка к действию Н-модели соответствует сдвигу температуры $\tau \rightarrow \tau-g_{2} \delta m$ в пренебрежении поправками от градиента $\delta m$. Поскольку в окрестности критической точки параметр $\tau$ - малая величина, малые добавки порядка $\delta m$ к ней существенны. Этим данное слагаемое (36) отличается от вклада $\partial\left(v_{\|} \psi\right)$, который в пренебрежении градиентом $\partial v_{\|}$является лишь малой поправкой $v_{\|} \partial \psi$ к “немалой” величине $v_{\perp} \partial \psi$ в Н-модели.

Из сказанного следует, что в рассматриваемой задаче точное выражение (36) можно заменить приближенным

$$
\delta S(\phi)=-\lambda_{11}^{\prime} g_{2} \psi^{\prime}\left(\partial^{2} \psi\right)(\delta m) .
$$

Здесь учитывается лишш эффект сдвига $\tau$, соответствуюший гармоническим колебаниям температуры в звуковой волне из-за процессов адиабатического расштрения и сжатия [4]. Он и является основным механизмом взаимодействия волны с критическими флуктуациями.

Подставив выражение $(36)$ с $\delta S(\phi)$ из (37) в (33) и отбросив несущественную для нашей задачи (см. выше) флуктуационную добавку, пропорциональную $\psi^{2}$, к источнику $\delta \eta_{2}$, получим следуюшую систему уравнений:

$$
\begin{aligned}
& \partial_{t}\left[\delta m(x)-\lambda_{11}^{\prime} A^{\prime} \psi^{2}(x) \int d x^{\prime} \psi^{\prime}\left(x^{\prime}\right) \partial^{2} \psi\left(x^{\prime}\right) \delta m\left(x^{\prime}\right)\right]+m_{0} \partial v_{\|}(x)=\delta \eta_{2}(x), \\
& \partial_{t} v_{\|}(x)+a^{-1} m_{0} \partial(\delta m(x))=\delta \eta_{3}(x), \quad A^{\prime} \equiv \frac{g_{2}^{2}}{2}
\end{aligned}
$$

Здесь указано явно подразумеваемое во всех формулах типа (37) интегрирование по $x \equiv(t, \mathbf{x})$.

Мы пришли к известной задаче о распространении волны в среде со случайными параметрами ("шумом"). В компактной форме система уравнений (38) для звуковых полей $\theta \equiv \delta m, v_{\|}$записывается в виде

$$
L \theta=J, \quad L=L_{0}-V,
$$

где $L_{0}$ - невозмушенный оператор, $V$ - возмушение, содержашее случайные поля $\psi, \psi^{\prime}$ с заданной (Н-моделью) функцией распределения. Задача (39) доопределяется естественным условием запаздывания. Нас интересует усредненное по распределению шума решение $\theta=L^{-1} J$ задачи (39), т.е. величина $\langle\theta\rangle=\langle G\rangle J, G \equiv L^{-1}$. По смыслу $\langle G\rangle=\left\langle L^{-1}\right\rangle$ - “полный пропагатор" волны. Его можно вычислить по теории возмущений, усредняя по распределению шума величину $G=L^{-1}=\left(L_{0}-V\right)^{-1}=G_{0}+$ $G_{0} V G_{0}+G_{0} V G_{0} V G_{0}+\ldots$ с $G_{0} \equiv L_{0}^{-1}$. Результат представляется диаграммами типа фейнмановских. Для полного пропагатора будет выполняться уравнение Дайсона

$$
\langle G\rangle^{-1}=L_{0}-\Sigma
$$


где

$$
\Sigma=\langle V\rangle+\left\langle V G_{0} V\right\rangle-\langle V\rangle G_{0}\langle V\rangle+\ldots
$$

есть сумма 1-неприводимых "собственно-энергетических" графов. Искомый закон дисперсии находится путем решения уравнения $\operatorname{det}\langle G\rangle^{-1}=\operatorname{det}\left[L_{0}-\Sigma\right]$ в импульсно-частотном представлении.

В нашем случае в (40) можно ограничиться простейшим приближением $\Sigma=\langle V\rangle$. Этот вклад определяется Н-моделью, а все следующие члены в разложении для $\Sigma$ дают лишь ИК-несущественные поправки от взаимодействия полей Н-модели со звуковыми модами. Это можно проверить явно оценкой вкладов второго порядка по $V$ в выражении (40) для $\Sigma$, представив их соответствуюшими фейнмановскими графами. УФ-("ультрафиолетово") расходяшиеся (что естественно для ИК-несушественных вставок) интегралы по импульсам внутренней звуковой линии в этих графах можно обрезать на флуктуационных масштабах $k_{\text {фл }} \sim \tau^{1 / 2}$, тогда ИК-несушественность данных вкладов становится очевидной. Таким образом, в нашей задаче с точностью до ИК-несущественных поправок

$$
\langle G\rangle^{-1}=\left\langle G^{-1}\right\rangle=\langle L\rangle
$$

т.е. усреднять по распределению шума можно сам оператор $L$ в (39), а не величину $G=$ $L^{-1}$. После усреднения $\langle\ldots\rangle$ вклад возмушения $V$ в уравнениях (38) представится в виде

$$
2 A^{\prime} \partial_{t} \int d x^{\prime} \bar{R}\left(x, x^{\prime}\right) \delta m\left(x^{\prime}\right)
$$

где $\bar{R}\left(x, x^{\prime}\right)$ - коррелятор двух составных операторов Н-модели:

$$
\begin{aligned}
& \bar{R}\left(x, x^{\prime}\right) \equiv\left\langle F(x) F^{\prime}\left(x^{\prime}\right)\right\rangle, \\
& F(x) \equiv \frac{\psi^{2}(x)-\left\langle\psi^{2}(x)\right\rangle}{2}, \quad F^{\prime}(x) \equiv-\lambda_{11}^{\prime} \psi^{\prime}(x) \partial^{2} \psi(x) .
\end{aligned}
$$

Вычитание константы $\left\langle\psi^{2}(x)\right\rangle$ из оператора $\psi^{2}(x)$ допустимо потому, что он входит в (38) в виде $\partial_{t} \psi^{2}(x)$, в операторы $F$ и $F^{\prime}$ коэффициенты введены для удобства. В импульсночастотном представлении свертка в (42) переходит в произведение $\bar{R}(\omega, k) \delta m(\omega, k)$, где $\omega$ и $k$ - параметры звуковой волны. Поскольку коррелятор $\bar{R}(\omega, k)$ определяется Н-моделью, "естественным масштабом" для его аргументов $\omega$ и $k$ являются соответствую-

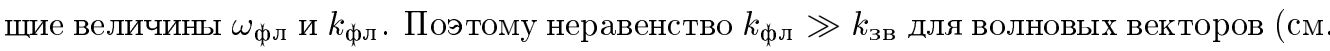
вьше) позволяет пренебречь зависимостью функции $\bar{R}(\omega, k)$ от $k$ и считать, что $k=0$. В таком приближении интеграл в (42) заменяется выражением

$$
\int d t^{\prime} R\left(t, t^{\prime}\right) \delta m\left(t^{\prime}, \mathbf{x}\right)
$$

где

$$
R\left(t, t^{\prime}\right) \equiv \int d \mathbf{x}^{\prime} \bar{R}\left(x, x^{\prime}\right) .
$$


Выражение (42) в приближении (44) в $\omega, k$-представлении принимает вид $-2 i \omega A^{\prime} R(\omega) \times$ $\delta m(\omega, k)$, поэтому для оператора $L$ в $(38)$ имеем

$$
\langle G\rangle^{-1}=\langle L\rangle=\left|\begin{array}{cc}
-i \omega\left[1+2 A^{\prime} R(\omega)\right] & m_{0} i k \\
a^{-1} m_{0} i k & -i \omega
\end{array}\right| .
$$

Отсюда получаем искомый закон дисперсии:

$$
k^{2}=\omega^{2}\left[c_{0}^{-2}+A_{0} R(\omega)\right], \quad A_{0} \equiv 2 A^{\prime} c_{0}^{-2},
$$

с неуниверсальными константами $c_{0}^{-2}$ из (34) и $A^{\prime}$ из (38).

При заданной частоте $\omega$ решение дисперсионного уравнения (46) для $k \equiv|k|$ ишется в виде $k=\omega c^{-1}+i \alpha$, откуда находятся положительные параметры $c$ (скорость звука) и $\alpha$ (коэффициент затухания). Обычно вместо $\alpha$ приводится величина $\alpha_{\lambda} \equiv \alpha \lambda_{3 в}=2 \pi c \alpha / \omega$ - затухание на длине волны $\lambda_{\text {зв }}$. В реальных условиях $\alpha \ll \omega / c$, и тогда из (46) следует, что

$$
c^{-2}(\omega)=c_{0}^{-2}+A_{0} \operatorname{Re} R(\omega), \quad \alpha_{\lambda}(\omega) c^{-2}(\omega)=\pi A_{0} \operatorname{Im} R(\omega) .
$$

Напомним, что $R(\omega)$ - фурье-образ коррелятора $R\left(t, t^{\prime}\right)$ из (44). По физическому смыслу этот коррелятор является функцией отклика величины $\langle F(x)\rangle$ на зависяшую только от времени вариацию температуры $\delta \tau\left(t^{\prime}\right)$ в Н-модели (с точностью до знака). Функция $R\left(t, t^{\prime}\right)$ не имеет $\delta$-образной особенности при $t=t^{\prime}$, поэтому ее фурье-образ $R(\omega)$ исчезает при $\omega \rightarrow \infty$, так что из (46) имеем

$$
R(\infty)=0, \quad c(\infty)=c_{0}, \quad \alpha_{\lambda}(\infty)=0 .
$$

Таким образом, константа $c_{0}$ имеет смысл предельной скорости звука при $\omega \rightarrow \infty$. Отметим, что эта величина не зависит от $\tau$ в отличие от $c(0)$. С точки зрения теории критического поведения параметры $c_{0}$ и $A_{0}$ в соотношениях (47) представляют собой не зависяшие от $\tau$ неуниверсальные константы. В правую часть второго равенства (47) параметр $c_{0}$ не входит, а из первого равенства (47) его можно исключить вычитанием величины $c^{-2}(\infty)=c_{0}^{-2}$.

Функцию отклика $R\left(t, t^{\prime}\right)$ можно выразить через коррелятор

$$
D\left(t, t^{\prime}\right) \equiv \int d \mathbf{x}^{\prime}\left\langle F(x) F\left(x^{\prime}\right)\right\rangle
$$

двух операторов $F$ из (43), воспользовавшись флуктуационно-диссипационной теоремой [12] для Н-модели:

$$
R\left(t, t^{\prime}\right)=\theta\left(t-t^{\prime}\right) \int d \mathbf{x}^{\prime}\left\langle F(x) \psi\left(x^{\prime}\right) \partial_{t^{\prime}} \psi\left(x^{\prime}\right)\right\rangle=\theta\left(t-t^{\prime}\right) \partial_{t^{\prime}} D\left(t, t^{\prime}\right) .
$$

Равенство (50) можно переписать в виде

$$
\begin{aligned}
& R\left(t, t^{\prime}\right)=\delta\left(t-t^{\prime}\right) D^{\mathrm{st}}+\partial_{t^{\prime}} D^{\mathrm{ret}}\left(t, t^{\prime}\right), \\
& D^{\mathrm{st}} \equiv D(t, t)=C, \quad D^{\mathrm{ret}}\left(t, t^{\prime}\right) \equiv \theta\left(t-t^{\prime}\right) D\left(t, t^{\prime}\right) .
\end{aligned}
$$


Согласно общим принципам соответствия между динамикой и статикой, коррелятор $D(t, t)$ не зависит от $t$ и совпадает с соответствуюшим статическим объектом, в данном случае имеюшим смысл теплоемкости $C=C_{v}$ в простой $\psi^{4}$-модели. В частотном представлении равенство (51) принимает следуюший вид:

$$
R(\omega)=D^{\mathrm{st}}+i \omega D^{\mathrm{ret}}(\omega), \quad D^{\mathrm{st}}=C .
$$

Соотношения (47), (48), (52) являются окончательными выражениями для закона дисперсии в рассматриваемой задаче. Они совпадают с результатами работы [10], отличаясь лишь способом вывода, в частности выбором динамических переменных. Однопетлевой расчет затухания и скорости звука на критической изохоре вблизи $T_{\mathrm{c}}$ был проделан в работе [9] (однако вывод общего закона дисперсии типа (46) в [9] некорректен). Результаты двухпетлевого расчета мы приведем в следующей работе.

Работа выполнена при поддержке РФФИ (грант № 96-02-17-033) и КЦФЕ Минобразования Р $\Phi$ (грант № 97-0-14.1-30).

\section{Список литературы}

[1] K. Kawasaki. Ann. Phys. N. Y. 1970. V. 61. P. 1.

[2] E.D. Siggia, B. I. Halperin, P. C. Hohenberg. Phys. Rev. B. 1976. V. 13. P. 2110.

[3] P. C. Hohenberg, B. I. Halperin. Rev. Mod. Phys. 1977. V. 49. P. 435.

[4] Л. Д. Ландау, Е. М. Лифииц. Гидродинамика. М.: Наука, 1986.

[5] А. З. Паташинский, В. Л. Покровский. Флуктуационная теория фазовых переходов. М.: Наука, 1982.

[6] Л. Д. Ландау, Е. М. Лифииц. Статистическая физика. Т.1 Изд. 3-е. М.: Наука, 1976.

[7] C. De Dominicis, L. Peliti. Phys. Rev. B. 1978. V. 18. P. 353.

[8] D. Roe, B. Wallace, H. Meyer. J. Low Temp. Phys. 1974. V. 16. P. 51; 1978. V. 30 . P. 91.

[9] D. M. Kroll, J. M. Ruhland. Phys. Lett. A. 1980. V. 80. P. 45.

[10] R. Dengler, F. Schwabl. Z. Phys. B. 1987. V. 69. P. 327.

[11] R. A. Ferrel, J. K. Bhattacharjee. Phys. Lett. A. 1981. V. 86. P. 109; 1982. V. 88. P. 77; Phys. Rev. A. 1985. V. 31. P. 1788; R. A. Ferrel, B. Mirhoshen, J. K. Bhattacharjee. Phys. Rev. B. 1987. V. 35. P. 4662.

[12] R. Baush, H. K. Janssen, H.Z. Wagner. Z. Phys. B. 1976. V. 24. P. 113. 